\title{
The Pattern of Cardiovascular Disease Risks among Working People (18-64 years) via a Nationwide Household-based Survey in Malaysia
}

\section{S Maria Awaluddin ( $\nabla$ smaria@moh.gov.my )}

National Institutes of Health, Ministry of Health Malaysia

Kuang Kuay Lim

National Institutes of Health, Ministry of Health Malaysia

Nor Syaqilah Shawaluddin

National Institutes of Health, Ministry of Health Malaysia

Tuan Amin Tuan Lah

National Institutes of Health, Ministry of Health Malaysia

Rosnawati Muhamad Robat

National Institutes of Health, Ministry of Health Malaysia

Maznieda Mahjom

National Institutes of Health, Ministry of Health Malaysia

Muhammad Fadhli Mohd Yussof

National Institutes of Health, Ministry of Health Malaysia

\section{Research Article}

Keywords: cardiovascular disease (CVD), occupation, industry classification, working, national health and morbidity survey (NHMS)

Posted Date: April 28th, 2022

DOI: https://doi.org/10.21203/rs.3.rs-1391598/v2

License: (c) (1) This work is licensed under a Creative Commons Attribution 4.0 International License. Read Full License 


\section{Abstract}

Background: Cardiovascular diseases (CVDs) are prevalent globally and are increasing tremendously. This article aimed to determine the prevalence and prevalence odds ratio of seven CVD risks among working people (18-64 years) in Malaysia.

Methods: A cross-sectional study among a nationally representative sample in Malaysia was conducted using a validated questionnaire. Clinical procedures were performed by registered nurses to measure glucose, cholesterol, body mass index and blood pressure. The prevalence and prevalence odds ratio were calculated via complex sample data analysis of IBM SPSS Statistics version 25.

Results: The overall prevalence of CVD risks among working people was $14.1 \%, 17.3 \%, 24.5 \%, 28.8 \%$, $31.7 \%, 45.9 \%$ and $94.2 \%$ for diabetes, obesity, hypertension, physical inactivity, tobacco smoking, hypercholesterolemia and inadequate fruit and vegetable (FV) intake, respectively. This study found that older age was associated with the odds of having the most CVD risks. Males were associated with higher odds of having hypertension and tobacco smoking, whereas females were more likely to have hypercholesterolemia, obesity, and physical inactivity. Those who worked as manager \& senior officials, clerical workers and services \& sales workers were physically inactive, while workers from the lower educational level were more likely to smoke tobacco. Workers in the public sector were more likely to have a higher prevalence of diabetes, hypertension, hypercholesterolemia, smoking and obesity than workers in the private sector.

Conclusions: This study highlighted that working people have significant CVD risks and a regular medical screening should be conducted at workplace settings.

\section{Introduction}

Cardiovascular disease (CVD) is a group of diseases, including ischemic heart disease, and stroke is the most common disease. The main target organs for CVD are the heart and blood vessels. The traditional risk of CVD is related to maladaptive behavior such as tobacco use, unhealthy diet, obesity, physical inactivity, and harmful alcohol use [1]. The global burden of diseases study in 1990-2019 listed ischemic heart disease as the top leading cause of disability-adjusted life-years (DALYs), followed by stroke and diabetes among people aged 50-74 years [2]. Malaysia is considered an upper-middle-income country and faces similar increasing CVD trends [3]. Therefore, the National Health and Morbidity Survey (NHMS) was conducted in Malaysia to monitor the trends of CVD risks among the Malaysian adult population every 4-year cycle. The NHMS estimates the prevalence of diabetes mellitus, hypertension, hypercholesterolemia, tobacco use, physical inactivity and obesity $[4,5]$.

CVD may cause premature death, and three-quarters of all premature deaths occur in low- and middleincome countries [1]. Thus, the recommendation of targeted CVD screening as early as 30 years was mentioned in the recent Malaysian Clinical Practices Guidelines on Primary \& Secondary Prevention of Cardiovascular Disease 2017 [6]. The age range of 18 to 64 years is the most productive age for working 
and maintaining their family's needs [7]. Unfortunately, younger adults were associated with low awareness of CVD risk, as found by previous local studies $[8,9]$.

Working-age refers to age 15 to 64 years in many countries, including Malaysia. In addition, there is a standard occupation classification according to the main job title and industry classification $[7,10]$. Occupation exposes people to occupational hazards that may have health effects in the long run, which mostly manifest after the individual has left the job [11, 12]. Ischemic heart disease remains the leading cause of mortality worldwide. Working people may have the uncovered burden of sudden CVD attacks due to a lack of awareness for early screening programs [13]. CVD is grouped under noncommunicable diseases (NCDs) and accounts for $38 \%$ of the total NCDs' premature mortality [14]. It is considered a work-related disease (WRD) due to indirect and multifactorial causes, such as sedentary lifestyle, sedentary work, hereditary factors and occupational characteristics [15]. The burden of CVD among workers was estimated from previous studies in certain occupations, such as mining workers [16, 17], public servants [18], healthcare workers [19], farmers [20], industrial sector workers [21] and community restaurants' food handlers [22]. Those who worked in the forest faced stressful working conditions with limited access to healthcare facilities [23], while truck drivers may have maladaptive behavior to meet their job demands [24].

The occupational characteristics in Malaysia were not explored in detail in previous NHMS, therefore a group of occupational experts proposed a new instrument for collecting data on occupational characteristics in NHMS 2015. This study aims to estimate the prevalence of seven types of diseases or risk factors that may contribute to CVD among working people in Malaysia. The second objective was to determine the prevalence odds ratio of each disease or risk factor that may contribute to CVD based on the sociodemographic profiles and occupational characteristics of working people. The diseases or risk factors that may contribute to CVD were diabetes mellitus, hypertension, hypercholesterolemia, obesity, smoking, physical inactivity and inadequate fruit and vegetable (FV) intake.

\section{Materials And Methods}

Data were obtained from the NHMS 2015, a scheduled nationwide household survey since 1986 . The data were used to review health priorities and plan the strategy and resources for delivering health care services, particularly NCD and its risk factors.

\section{Population and sampling}

The NHMS used a national representative sample; hence, multistage random cluster sampling was applied to cover urban and rural areas in all 13 states and three federal territories (FT) in Malaysia. Malaysia's geographical areas were divided into enumeration blocks (EB) with 80 to 120 living quarters (LQs) per EB. It may also consist of an average of 500 to 600 people. The Department of Statistics Malaysia provided the sampling frame. The primary sampling unit was the EBs, and the LQs were the secondary sampling unit. All households in the selected LQs and members in the household were eligible 
to participate in this study. The NHMS 2015 required 10,428 LQs to meet the core objectives based on a single proportion formula for prevalence estimation. A few adjustments were made to the total sample size considering the design effect and non-response rate of 35\%. Urban and rural areas were taken into consideration and were calculated proportionally to population size. The NHMS utilized a cross-sectional study design and covered all age groups. Further details of the methodology of this study have been described in the technical report [5]. This analysis focused on the respondents who answered the question "Are you working?" referring to the past month among respondents aged 18 to 64 years. The defined age for the labour force in Malaysia was 15-64 years [25]. However, the age of 18 years was taken because the CVD burdens were only measured for adults aged 18 years and above in the NHMS survey.

\section{Instruments and training}

This survey used a validated questionnaire and a standard clinical procedure of point of care testing. A new questionnaire for occupational characteristics was incorporated into this NHMS cycle. The questionnaire was developed by a panel of experts in occupational health and underwent a pretest session. The main job title was based on the Malaysia Standard Classification of Occupations (MASCO) 2013 , and it has ten groups of main job titles [10]. The industry classification was adapted from Malaysia Standard Industrial Classification (MISC) 2008 from the alphabet A to $U$ classification groups [25]. The process of developing occupational characteristics questionnaires and the occupational questionnaire and original local classifications are attached in Supplement 1. The second part was the clinical measurement, which included anthropometric measurements for weight and height. A capillary blood sampling was perfomed to measure fasting blood glucose and cholesterol. The Tanita Personal Scale HD 319, SECA Stadiometer 213 and CardioChek ${ }^{\circledR}$ PA Analyzer were utilized in this survey. Blood pressure was measured using the Omron Japan Model HEM-907. Before the actual survey, a one-week formal data collection training was held in Peninsular Malaysia and Sabah \& Sarawak. The data collection team consisted of temporary personnel who conducted the interviews while the permanent staff nurses undertook the clinical procedures. Adherence to the safety procedure and standard clinical waste disposal were ensured.

\section{Variable definitions}

The CVD risks measured in this study were the prevalence of overall diabetes, hypertension and hypercholesterolemia. It consisted of those who were newly diagnosed during the survey and those who were previously diagnosed by medical staff. In addition, current tobacco smokers, physical inactivity, obesity and inadequate FV intake were included. The sociodemographic variables were age group, gender and education level. The working status was elaborated in terms of main job title, industrial classification, job sector, working time and duration of working. The age of working adults was further categorized into three age groups of $18-34,35-49$ and 50 years and above as used by other studies [26, 27]. The definition of all variables measured in this study was according to the standard guidelines and can be referred to in the NMHS 2015 technical reports, which are publicly accessible via this link http://iku.gov.my/nhms-2015 [4, 5]. 


\section{Ethical approval and consent to participate}

The ethical clearance was granted from the Medical Research and Ethics Committee (MREC), Ministry of Health and this survey was registered with the National Medical Research Registration with NMRR ID-141064-21877. Written consent was obtained from each individual respondent prior to data collection. This study was conducted in accordance with the Declaration of Helsinki.

\section{Statistical analysis}

IBM SPSS Statistics for Windows, Version 25 software was used to calculate the prevalence of CVD risks among the Malaysian working persosn via the complex sample design. A weightage was applied in the data analysis due to multiple stages of sampling selection, such as EBs and LQ selection. In addition, the weightage calculation also considered the non-response rate and population factors such as age, gender, ethnicity, strata and state. By applying weight, this study produces a better population estimation and inferences. The findings were tabulated in a table consisting of an unweighted count, weighted prevalence, $95 \%$ confidence interval $(\mathrm{Cl})$ and estimated population. Finally, the prevalence odds ratio (POR) was calculated using multivariable logistic regression. An odds ratio $>1$ was defined as having higher risk, while an odds ratio $<1$ was defined as having low risk. A p-value of $<0.05$ was considered statistically significant.

\section{Results}

The overall response rate for the national representative of the NHMS household survey was $86.4 \%$ from the total 9,433 eligible LQs and 30,548 individual respondents. This study focused on analyzing 11,543 respondents aged 18 to 64 years who were working during the survey duration. The figure can be further estimated to be 12.9 million people and it may represent the total working population in Malaysia. More than half of the respondents (52.1\%) were 18 to 34 years old. Of the total respondents, $61.6 \%$ were males, and only $21.7 \%$ of the total respondents resided in urban areas. The occupational characteristics are shown in Table 1 in terms of the main job title, industry classification, working time system and total duration of work. Approximately $60 \%$ of the respondents were from private sectors, $14.8 \%$ were from government sectors, and the remaining respondents were reported as self-employed. The main job title showed that one-third were general workers or unskilled groups, and only $4.8 \%$ were managers and senior officials. The top three industries with the highest proportion of workers were manufacturing, wholesale and retail trade and the repair of motor vehicles and motorcycles, followed by agriculture and forestry and fishing, at $12.2 \%, 12.1 \%$, and $10.1 \%$, respectively. According to the new instrument used in this study, $14.6 \%$ of the respondents worked in industries other than the 12 listed industries. 
Table 1

The sociodemography of working people (18-64 years) in NHMS 2015; $n=11,543$

\begin{tabular}{|c|c|c|c|}
\hline Variables & $\begin{array}{l}\text { Unweighted } \\
\text { count }\end{array}$ & $\begin{array}{l}\text { Weighted \% } \\
(95 \% \mathrm{Cl})\end{array}$ & $\begin{array}{l}\text { Estimated } \\
\text { population }\end{array}$ \\
\hline \multicolumn{4}{|l|}{ Age group (years) } \\
\hline $18-34$ & 4,880 & $\begin{array}{l}50.6(49.1 \\
52.1)\end{array}$ & $6,508,718$ \\
\hline $35-49$ & 4,164 & $\begin{array}{l}33.9(32.5, \\
35.3)\end{array}$ & $4,355,816$ \\
\hline 50 and above & 2,499 & $\begin{array}{l}15.5(14.6 \\
14.6)\end{array}$ & $1,996,088$ \\
\hline \multicolumn{4}{|l|}{ Gender } \\
\hline Male & 6,922 & $\begin{array}{l}62.8(61.6, \\
63.9)\end{array}$ & $8,072,641$ \\
\hline Female & 4,621 & $\begin{array}{l}37.2(36.1 \\
38.4)\end{array}$ & $4,787,980$ \\
\hline \multicolumn{4}{|l|}{ Strata } \\
\hline Urban & 4,406 & $\begin{array}{l}21.7(20.4, \\
23.0)\end{array}$ & $2,788,707$ \\
\hline Rural & 7,137 & $\begin{array}{l}78.3(77.0, \\
79.6)\end{array}$ & $10,071,914$ \\
\hline \multicolumn{4}{|l|}{ Level of education } \\
\hline Primary & 3,394 & $\begin{array}{l}31.7(29.9 \\
33.5)\end{array}$ & $4,068,718$ \\
\hline Secondary & 5,682 & $\begin{array}{l}47.4(45.8, \\
49.0)\end{array}$ & $6,090,628$ \\
\hline Tertiary & 2,458 & $\begin{array}{l}20.9 \\
22.7)\end{array}$ & $2,691,299$ \\
\hline \multicolumn{4}{|l|}{ Main job title classification (MASCO 2013) } \\
\hline G1. Managers \& senior officials & 464 & $\begin{array}{l}4.8(4.2 \\
5.5)\end{array}$ & 611,904 \\
\hline G2. Professionals & 1,394 & $\begin{array}{l}11.8(10.8 \\
13.0)\end{array}$ & $1,515,814$ \\
\hline G3. Technician \& associate professionals & 588 & $\begin{array}{l}5.5(4.9 \\
6.1)\end{array}$ & 700,968 \\
\hline G4. Clerical workers & 935 & $\begin{array}{l}7.5(6.8, \\
8.2)\end{array}$ & 963,861 \\
\hline
\end{tabular}




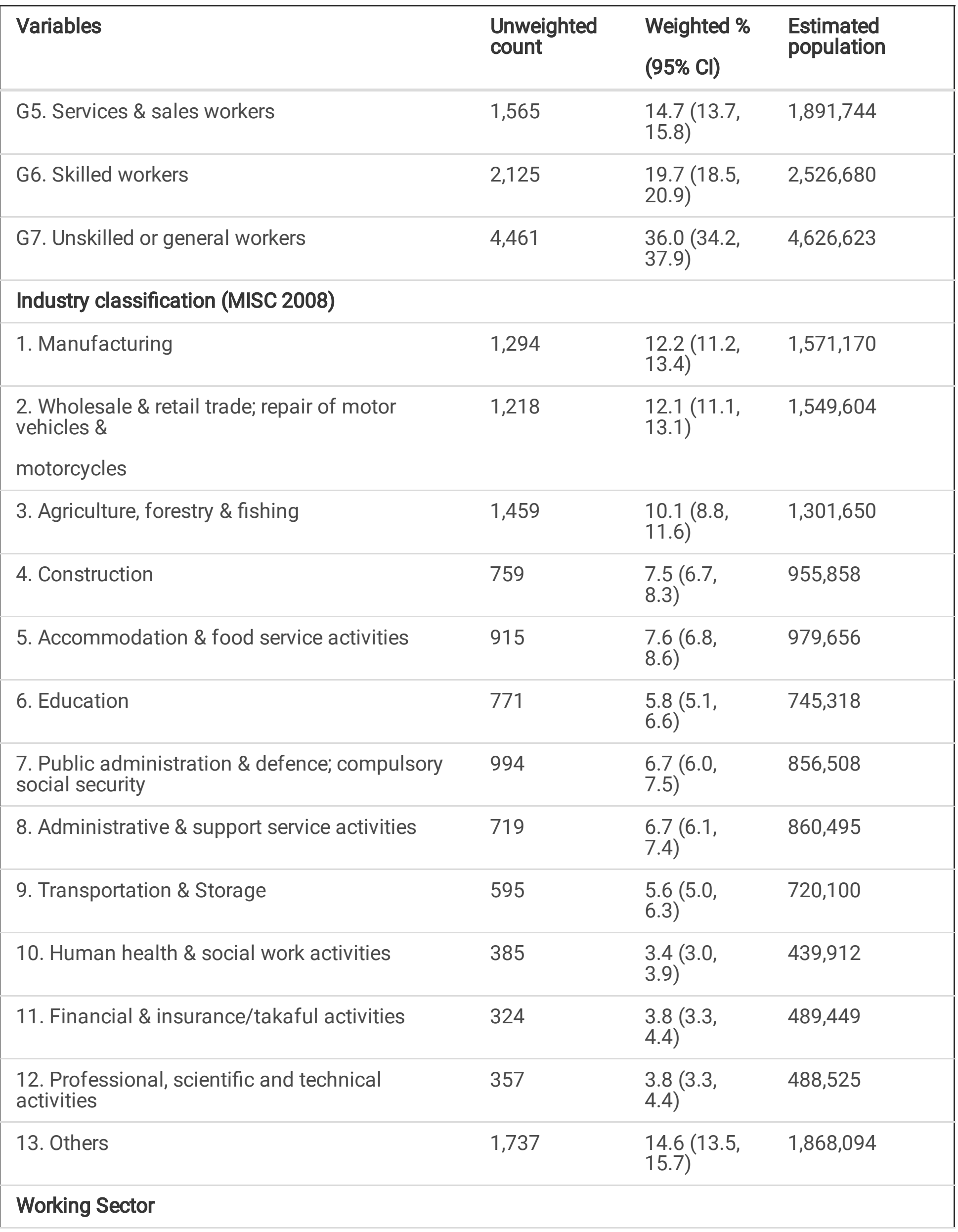




\begin{tabular}{|c|c|c|c|}
\hline Variables & $\begin{array}{l}\text { Unweighted } \\
\text { count }\end{array}$ & $\begin{array}{l}\text { Weighted \% } \\
(95 \% \mathrm{Cl})\end{array}$ & $\begin{array}{l}\text { Estimated } \\
\text { population }\end{array}$ \\
\hline Government \& semigovernment & 2,167 & $\begin{array}{l}14.8(13.5, \\
16.2)\end{array}$ & $1,903,681$ \\
\hline Private & 5,934 & $\begin{array}{l}60.7(58.8, \\
62.6)\end{array}$ & $7,808,584$ \\
\hline Self-employed & 3,441 & $\begin{array}{l}24.5(23.1, \\
25.9)\end{array}$ & $3,147,856$ \\
\hline Working in more than one sector & 298 & $\begin{array}{l}2.8(2.3 \\
3.3)\end{array}$ & 355,261 \\
\hline \multicolumn{4}{|l|}{ Working time system } \\
\hline Regular working time & 5,950 & $\begin{array}{l}53.2(51.7 \\
54.8)\end{array}$ & $6,836,805$ \\
\hline Extended hours & 1,235 & $\begin{array}{l}12.1(11.1 \\
13.2)\end{array}$ & $1,556,066$ \\
\hline Staggered days & 3,015 & $\begin{array}{l}22.9(21.6, \\
24.3)\end{array}$ & $2,945,103$ \\
\hline Shift hours & 1,147 & $\begin{array}{l}10.2(9.1 \\
11.4)\end{array}$ & $1,308,739$ \\
\hline Long haul & 28 & $\begin{array}{l}0.2(0.1 \\
0.4)\end{array}$ & 29,186 \\
\hline Part-time & 157 & $\begin{array}{l}1.3(1.0, \\
1.6)\end{array}$ & 167,628 \\
\hline Mean duration of work (years) & - & $\begin{array}{l}9.0(8.7 \\
9.2)\end{array}$ & - \\
\hline \multicolumn{4}{|l|}{ Total duration of work (years) } \\
\hline 5 and below & 5,219 & $\begin{array}{l}50.3(48.7, \\
51.8)\end{array}$ & $6,419,471$ \\
\hline 6 to 10 & 2,373 & $\begin{array}{l}20.1(19.1, \\
21.2)\end{array}$ & $2,571,445$ \\
\hline$>10$ years & 3,882 & $\begin{array}{l}29.6(28.3 \\
30.9)\end{array}$ & $3,782,051$ \\
\hline
\end{tabular}

Table 2 shows the distribution of overall burden of CVD risks among working people in Malaysia. The prevalence was $14.1 \%$; (95\% Cl: 13.1, 15.0), 24.5\%; (95\% Cl: 23.4, 25.6), 45.9\%; (95\% Cl: 44.4, 47.4), and 17.3\%; (95\% Cl: $16.3,18.3)$, for diabetes, hypertension, hypercholesterolemia and obesity, respectively. The prevalence of diabetes is nearly equal between the sexes. Males had a higher prevalence of hypertension 
and were current tobacco smokers. In comparison, females had a higher prevalence of hypercholesterolemia and obesity and were physically inactive than males. In terms of age groups, younger adults had a higher prevalence of current tobacco smokers and inadequate FV intake, 32.8\% (95\% Cl: 30.8, 34.8), 95.0\% (95\% Cl: 93.9, 95.9), respectively. Older adults had a higher prevalence of diabetes mellitus (27.8\%; $95 \%$ Cl: $25.4,30.2)$, hypertension (49.1\%; $95 \% \mathrm{Cl}: 46.5,51.7)$ and hypercholesterolemia $(64.5 \%$; $95 \% \mathrm{Cl}: 61.8,67.1)$. The CVD risks based on the main job title highlighted that the managers and senior officials had a higher prevalence of hypercholesterolemia $(50.7 \% ; 95 \% \mathrm{Cl}$ : $44.7,56.7)$, the skilled worker group had a higher prevalence of smoking (60.1\%; 95\% Cl: 57.0, 63.1), while obesity and physical inactivity were prevalent among those in the administrative work or white-collar group. By industry classification, diabetes was the highest among those who work in transportation \& storage; hypertension was the highest among public administration \& defense staff; compulsory social security and inadequate FV intake among construction workers. The prevalence of hypercholesterolemia was the highest among those in the education industry. The transportation and storage industry has a higher prevalence of multiple CVD risks, including diabetes, hypertension, smoking, obesity and inadequate FV intake. 
Table 2

The prevalence of CVD risks among working people via household survey in Malaysia.

\begin{tabular}{|c|c|c|c|}
\hline Variables & $\begin{array}{l}\text { Diabetes } \\
\text { mellitus }\end{array}$ & Hypertension & Hypercholesterolemia \\
\hline & $\%(95 \% \mathrm{Cl})$ & $\%(95 \% \mathrm{Cl})$ & $\%(95 \% \mathrm{Cl})$ \\
\hline Overall & $\begin{array}{l}\text { 14.1(13.1, } \\
15.0)\end{array}$ & $\begin{array}{l}24.5(23.4 \\
25.6)\end{array}$ & $45.9(44.4,47.4)$ \\
\hline \multicolumn{4}{|l|}{ Age group (years) } \\
\hline $18-34$ & $8.4(7.4,9.5)$ & $\begin{array}{l}13.2(11.9 \\
14.5)\end{array}$ & $34.0(32.1,35.9)$ \\
\hline $35-49$ & $\begin{array}{l}16.3(14.7 \\
17.9)\end{array}$ & $\begin{array}{l}30.1(28.4, \\
32.0)\end{array}$ & $55.2(52.9,57.5)$ \\
\hline 50 and above & $\begin{array}{l}27.8(25.4 \\
30.2)\end{array}$ & $\begin{array}{l}49.1(46.5, \\
51.7)\end{array}$ & $64.5(61.8,67.1)$ \\
\hline \multicolumn{4}{|l|}{ Gender } \\
\hline Male & $\begin{array}{l}14.5(13.3 \\
15.6)\end{array}$ & $\begin{array}{l}26.8(25.4, \\
28.3)\end{array}$ & $44.0(42.3,45.8)$ \\
\hline Female & $\begin{array}{l}13.4(12.1 \\
14.8)\end{array}$ & $\begin{array}{l}20.5(19.1 \\
22.0)\end{array}$ & $49.1(46.9,51.2)$ \\
\hline \multicolumn{4}{|l|}{ Level of education } \\
\hline Primary & $\begin{array}{l}17.0(15.0 \\
19.3)\end{array}$ & $\begin{array}{l}31.2(28.4, \\
34.1)\end{array}$ & $47.3(44.2,50.3)$ \\
\hline Secondary & $\begin{array}{l}14.3(13.1 \\
15.7)\end{array}$ & $\begin{array}{l}24.2(22.7 \\
25.7)\end{array}$ & $45.6(43.7,47.5)$ \\
\hline Tertiary & $\begin{array}{l}11.7(10.3 \\
13.3)\end{array}$ & $\begin{array}{l}20.5(18.7 \\
22.5)\end{array}$ & $45.5(43.1,48.0)$ \\
\hline \multicolumn{4}{|l|}{ Main job title classification (MASCO 2013) } \\
\hline G1. Managers \& senior officials & $\begin{array}{l}12.5(9.0 \\
17.0)\end{array}$ & $\begin{array}{l}23.8(19.2, \\
29.1)\end{array}$ & $50.7(44.7,56.7)$ \\
\hline G2. Professionals & $\begin{array}{l}14.6(12.2, \\
17.3)\end{array}$ & $\begin{array}{l}23.6(20.9, \\
26.4)\end{array}$ & $50.6(47.0,54.1)$ \\
\hline G3. Technician \& associate professionals & $\begin{array}{l}14.1(11.1, \\
17.8)\end{array}$ & $\begin{array}{l}25.1(20.9, \\
29.8)\end{array}$ & $46.2(40.9,51.5)$ \\
\hline G4. Clerical workers & $\begin{array}{l}12.4(9.9 \\
15.5)\end{array}$ & $\begin{array}{l}20.7(17.7 \\
24.1)\end{array}$ & $43.0(39.0,47.0)$ \\
\hline G5. Services \& sales workers & $\begin{array}{l}11.4(9.5 \\
13.5)\end{array}$ & $\begin{array}{l}22.9 \\
25.5)\end{array}$ & $43.1(40.0,46.3)$ \\
\hline
\end{tabular}




\begin{tabular}{|c|c|c|c|}
\hline Variables & $\begin{array}{l}\text { Diabetes } \\
\text { mellitus }\end{array}$ & Hypertension & Hypercholesterolemia \\
\hline G6. Skilled workers & $\begin{array}{l}14.9(13.2 \\
16.9)\end{array}$ & $\begin{array}{l}25.1(22.8 \\
27.5)\end{array}$ & $46.5(43.5,49.7)$ \\
\hline G7. Unskilled or general workers & $\begin{array}{l}15.1(13.6, \\
16.7)\end{array}$ & $\begin{array}{l}25.9(24.1 \\
27.8)\end{array}$ & $45.0(43.0,47.1)$ \\
\hline \multicolumn{4}{|l|}{ Industry classification (MISC 2008) } \\
\hline 1. Manufacturing & $\begin{array}{l}14.7(12.2 \\
17.6)\end{array}$ & $\begin{array}{l}21.9(19.0 \\
25.0)\end{array}$ & $44.4(40.7,48.1)$ \\
\hline $\begin{array}{l}\text { 2. Wholesale \& retail trade; repair of motor } \\
\text { vehicles \& }\end{array}$ & $\begin{array}{l}11.1(9.3 \\
13.2)\end{array}$ & $\begin{array}{l}24.6(21.4 \\
28.0)\end{array}$ & $37.5(34.2,41.0)$ \\
\hline \multicolumn{4}{|l|}{ motorcycles } \\
\hline 3. Agriculture, forestry \& fishing & $\begin{array}{l}13.9(11.5, \\
16.6)\end{array}$ & $\begin{array}{l}28.9(25.5 \\
32.7)\end{array}$ & $46.3(42.6,50.0)$ \\
\hline 4. Construction & $\begin{array}{l}12.5(9.8 \\
15.9)\end{array}$ & $\begin{array}{l}23.5(19.4 \\
28.0)\end{array}$ & $43.7(39.1,48.5)$ \\
\hline 5. Accommodation \& food service activities & $\begin{array}{l}16.2(13.2 \\
19.7)\end{array}$ & $\begin{array}{l}25.1(21.9 \\
28.6)\end{array}$ & $49.3(45.2,53.4)$ \\
\hline 6. Education & $\begin{array}{l}17.7(14.5, \\
21.5)\end{array}$ & $\begin{array}{l}26.0(22.5 \\
29.9)\end{array}$ & $55.1(50.3,59.8)$ \\
\hline $\begin{array}{l}\text { 7. Public administration \& defence; } \\
\text { compulsory social security }\end{array}$ & $\begin{array}{l}15.5(12.9 \\
18.4)\end{array}$ & $\begin{array}{l}29.0(25.4 \\
32.8)\end{array}$ & $49.8(45.5,54.1)$ \\
\hline $\begin{array}{l}\text { 8. Administrative \& support service } \\
\text { activities }\end{array}$ & $\begin{array}{l}11.2(8.7 \\
14.4)\end{array}$ & $\begin{array}{l}19.4(16.2 \\
23.1)\end{array}$ & $43.1(38.6,47.6)$ \\
\hline 9. Transportation \& Storage & $\begin{array}{l}19.1(15.1 \\
23.9)\end{array}$ & $\begin{array}{l}27.6(23.3 \\
32.3)\end{array}$ & $47.9(42.3,53.5)$ \\
\hline 10. Human health \& social work activities & $\begin{array}{l}13.5(9.9 \\
18.3)\end{array}$ & $\begin{array}{l}25.3(20.0 \\
31.4)\end{array}$ & $54.0(47.5,60.4)$ \\
\hline 11. Financial \& insurance/takaful activities & $\begin{array}{l}12.4(8.2 \\
18.2)\end{array}$ & $\begin{array}{l}23.7(18.9 \\
29.3)\end{array}$ & $47.4(39.6,55.3)$ \\
\hline $\begin{array}{l}\text { 12. Professional, scientific and technical } \\
\text { activities }\end{array}$ & $\begin{array}{l}11.7(8.5 \\
15.8)\end{array}$ & $\begin{array}{l}19.8(15.3 \\
25.2)\end{array}$ & $45.4(39.2,51.7)$ \\
\hline 13. Others & $\begin{array}{l}14.3(12.3 \\
16.5)\end{array}$ & $\begin{array}{l}23.6(21.1 \\
26.2)\end{array}$ & $46.1(42.9,49.3)$ \\
\hline \multicolumn{4}{|l|}{ Working Sector } \\
\hline Government \& semigovernment & $\begin{array}{l}17.2(15.1, \\
19.5)\end{array}$ & $\begin{array}{l}29.5(27.0 \\
32.2)\end{array}$ & $54.4(51.2,57.6)$ \\
\hline
\end{tabular}




\begin{tabular}{|llll|}
\hline Variables & $\begin{array}{l}\text { Diabetes } \\
\text { mellitus }\end{array}$ & Hypertension & Hypercholesterolemia \\
\hline Private & $\begin{array}{l}12.0(10.9, \\
13.2)\end{array}$ & $\begin{array}{l}21.3(19.9, \\
22.8)\end{array}$ & $42.6(40.8,44.5)$ \\
\hline Self-employed & $\begin{array}{l}17.2(15.5, \\
19.1)\end{array}$ & $\begin{array}{l}29.3(27.2, \\
31.4)\end{array}$ & $48.9(46.5,51.3)$ \\
\hline Working time system & & & \\
\hline Regular working time & $14.1(12.9$, & $24.0(22.6$, & $47.0(45.1,49.0)$ \\
& $15.4)$ & $25.4)$ & \\
\hline Extended hours & $13.2(11.2$, & $23.3(20.2$, & $42.4(38.8,46.0)$ \\
& $15.6)$ & $26.8)$ & \\
\hline Staggered days & $16.1(14.4$, & $29.3(27.0$, & $47.6(45.0,50.2)$ \\
& $17.9)$ & $31.6)$ & \\
\hline Shift hours \& part-time & $10.9(8.9$, & $18.8(16.4$, & $41.0(37.7,44.3)$ \\
\hline
\end{tabular}


Table 2

The prevalence of CVD risks among working people via household survey in Malaysia (cont'd).

\begin{tabular}{|c|c|c|c|c|}
\hline Variables & Smoking & $\begin{array}{l}\mathrm{BMI} \geq 30 \\
\mathrm{~kg} / \mathrm{m}^{2}\end{array}$ & Inactive & $\begin{array}{l}\text { Inadequate } \\
\text { FV intake }\end{array}$ \\
\hline & $\begin{array}{l}\%(95 \% \\
\mathrm{Cl})\end{array}$ & $\begin{array}{l}\%(95 \% \\
\mathrm{Cl})\end{array}$ & $\begin{array}{l}\%(95 \% \\
\mathrm{Cl})\end{array}$ & $\%(95 \% \mathrm{Cl})$ \\
\hline Overall & $\begin{array}{l}31.7 \\
(30.3 \\
33.1)\end{array}$ & $\begin{array}{l}17.3 \\
(16.3 \\
18.3)\end{array}$ & $\begin{array}{l}28.3 \\
(27.1, \\
29.6)\end{array}$ & $\begin{array}{l}94.2(93.4, \\
94.9)\end{array}$ \\
\hline \multicolumn{5}{|l|}{ Age group (years) } \\
\hline $18-34$ & $\begin{array}{l}32.8 \\
(30.8, \\
34.8)\end{array}$ & $\begin{array}{l}15.7 \\
(14.4, \\
17.1)\end{array}$ & $\begin{array}{l}29.3 \\
(27.6 \\
31.1)\end{array}$ & $\begin{array}{l}95.0(93.9, \\
95.9)\end{array}$ \\
\hline $35-49$ & $\begin{array}{l}32.5 \\
(30.5 \\
34.6)\end{array}$ & $\begin{array}{l}19.1 \\
(17.6 \\
20.8)\end{array}$ & $\begin{array}{l}26.7 \\
(24.9 \\
28.7)\end{array}$ & $\begin{array}{l}93.9(92.8, \\
94.9)\end{array}$ \\
\hline 50 and above & $\begin{array}{l}26.1 \\
(24.0 \\
28.4)\end{array}$ & $\begin{array}{l}18.1(16.2, \\
20.2)\end{array}$ & $\begin{array}{l}28.5 \\
(26.2 \\
31.0)\end{array}$ & $\begin{array}{l}92.4(90.6, \\
93.9)\end{array}$ \\
\hline \multicolumn{5}{|l|}{ Gender } \\
\hline Male & $\begin{array}{l}49.4 \\
(47.6 \\
51.3)\end{array}$ & $\begin{array}{l}15.4 \\
(14.2 \\
16.7)\end{array}$ & $\begin{array}{l}24.5 \\
(23.1, \\
26.0)\end{array}$ & $\begin{array}{l}94.5(93.6, \\
95.3)\end{array}$ \\
\hline Female & $\begin{array}{l}1.7(1.2 \\
2.5)\end{array}$ & $\begin{array}{l}20.5 \\
(18.9 \\
22.1)\end{array}$ & $\begin{array}{l}34.7 \\
(32.9 \\
36.7)\end{array}$ & $\begin{array}{l}93.8(92.7, \\
94.7)\end{array}$ \\
\hline \multicolumn{5}{|l|}{ Level of education } \\
\hline Primary & $\begin{array}{l}41.9 \\
(38.9 \\
45.0)\end{array}$ & $\begin{array}{l}12.8 \\
(11.1, \\
14.9)^{\prime}\end{array}$ & $\begin{array}{l}20.4 \\
(18.4 \\
22.6)\end{array}$ & $\begin{array}{l}95.4(94.2, \\
96.3)\end{array}$ \\
\hline Secondary & $\begin{array}{l}37.0 \\
(35.2 \\
39.0)\end{array}$ & $\begin{array}{l}18.7 \\
(17.4 \\
20.0)\end{array}$ & $\begin{array}{l}27.0 \\
(25.4 \\
28.8)\end{array}$ & $\begin{array}{l}94.3(93.3, \\
95.2)\end{array}$ \\
\hline Tertiary & $\begin{array}{l}16.8 \\
(15.3 \\
18.5)\end{array}$ & $\begin{array}{l}18.2 \\
(16.5 \\
20.0)\end{array}$ & $\begin{array}{l}35.4 \\
(33.2 \\
37.5)\end{array}$ & $\begin{array}{l}93.3(91.8, \\
94.5)\end{array}$ \\
\hline \multicolumn{5}{|c|}{ Main job title classification (MASCO 2013) } \\
\hline G1. Managers \& senior officials & $\begin{array}{l}23.7 \\
(19.1 \\
28.9)^{\prime}\end{array}$ & $\begin{array}{l}19.4 \\
(15.6 \\
23.9)\end{array}$ & $\begin{array}{l}39.0 \\
(33.9 \\
44.4)^{\prime}\end{array}$ & $\begin{array}{l}92.5(89.3 \\
94.9)\end{array}$ \\
\hline
\end{tabular}




\begin{tabular}{|c|c|c|c|c|}
\hline Variables & Smoking & $\begin{array}{l}\mathrm{BMI} \geq 30 \\
\mathrm{~kg} / \mathrm{m}^{2}\end{array}$ & Inactive & $\begin{array}{l}\text { Inadequate } \\
\text { FV intake }\end{array}$ \\
\hline G2. Professionals & $\begin{array}{l}14.6 \\
(12.4 \\
17.2)\end{array}$ & $\begin{array}{l}20.8 \\
(18.1 \\
23.9)\end{array}$ & $\begin{array}{l}36.2 \\
(32.9 \\
39.6)\end{array}$ & $\begin{array}{l}92.0(89.8, \\
93.8)\end{array}$ \\
\hline G3. Technician \& associate professionals & $\begin{array}{l}32.8 \\
(28.1 \\
37.8)\end{array}$ & $\begin{array}{l}17.7 \\
(14.1 \\
22.0)\end{array}$ & $\begin{array}{l}30.2 \\
(25.6 \\
35.3)\end{array}$ & $\begin{array}{l}94.2(91.1 \\
96.3)\end{array}$ \\
\hline G4. Clerical workers & $\begin{array}{l}9.3(7.2 \\
11.9)\end{array}$ & $\begin{array}{l}20.4 \\
(17.3 \\
23.8)\end{array}$ & $\begin{array}{l}39.7 \\
(35.5, \\
44.0)\end{array}$ & $\begin{array}{l}94.4(92.2, \\
96.1)\end{array}$ \\
\hline G5. Services \& sales workers & $\begin{array}{l}22.5 \\
(19.8 \\
25.4)\end{array}$ & $\begin{array}{l}17.1 \\
(14.8, \\
19.7)\end{array}$ & $\begin{array}{l}31.6 \\
(28.5 \\
34.8)\end{array}$ & $\begin{array}{l}93.4(91.3 \\
95.0)\end{array}$ \\
\hline G6. Skilled workers & $\begin{array}{l}60.1 \\
(57.0 \\
63.1)\end{array}$ & $\begin{array}{l}17.3 \\
(15.3 \\
19.5)\end{array}$ & $\begin{array}{l}24.8 \\
(22.5 \\
27.4)\end{array}$ & $\begin{array}{l}94.6(93.3 \\
95.8)\end{array}$ \\
\hline G7. Unskilled or general workers & $\begin{array}{l}42.0 \\
(40.0 \\
44.1)\end{array}$ & $\begin{array}{l}15.3 \\
(13.9 \\
16.8)\end{array}$ & $\begin{array}{l}22.0 \\
(20.3 \\
23.8)\end{array}$ & $\begin{array}{l}95.2(94.2 \\
96.1)\end{array}$ \\
\hline \multicolumn{5}{|l|}{ Industry classification (MISC 2008) } \\
\hline 1. Manufacturing & $\begin{array}{l}34.7 \\
(31.3, \\
38.2)\end{array}$ & $\begin{array}{l}15.1 \\
(12.7 \\
17.8)\end{array}$ & $\begin{array}{l}24.7 \\
(21.7 \\
28.0)\end{array}$ & $\begin{array}{l}94.9(93.1 \\
96.2)\end{array}$ \\
\hline $\begin{array}{l}\text { 2. Wholesale } \& \text { retail trade; repair of motor } \\
\text { vehicles \& motorcycles }\end{array}$ & $\begin{array}{l}28.6 \\
(25.5 \\
31.9)\end{array}$ & $\begin{array}{l}13.7 \\
(11.6, \\
16.1)\end{array}$ & $\begin{array}{l}32.0 \\
(28.4, \\
35.8)\end{array}$ & $\begin{array}{l}93.2(91.2 \\
94.8)\end{array}$ \\
\hline 3. Agriculture, forestry \& fishing & $\begin{array}{l}49.8 \\
(46.1 \\
53.6)\end{array}$ & $\begin{array}{l}11.9(9.7 \\
14.5)\end{array}$ & $\begin{array}{l}12.0(9.9 \\
14.4)\end{array}$ & $\begin{array}{l}93.1(91.0 \\
94.8)\end{array}$ \\
\hline 4. Construction & $\begin{array}{l}51.6 \\
(46.4 \\
56.9)\end{array}$ & $\begin{array}{l}12.9 \\
(10.1, \\
16.5)^{\prime}\end{array}$ & $\begin{array}{l}17.4 \\
(14.1 \\
21.3)\end{array}$ & $\begin{array}{l}96.6(94.8 \\
97.7)\end{array}$ \\
\hline 5. Accommodation \& food service activities & $\begin{array}{l}28.1 \\
(23.5, \\
33.1)\end{array}$ & $\begin{array}{l}19.8 \\
(16.5, \\
23.6)\end{array}$ & $\begin{array}{l}24.3 \\
(20.8 \\
28.3)\end{array}$ & $\begin{array}{l}94.0(91.7 \\
95.6)\end{array}$ \\
\hline 6. Education & $\begin{array}{l}6.2(4.2 \\
9.1)\end{array}$ & $\begin{array}{l}24.9 \\
(21.0 \\
29.2)\end{array}$ & $\begin{array}{l}36.5 \\
(32.3 \\
40.9)\end{array}$ & $\begin{array}{l}90.0(85.8, \\
93.0)\end{array}$ \\
\hline $\begin{array}{l}\text { 7. Public administration \& defence; } \\
\text { compulsory social security }\end{array}$ & $\begin{array}{l}33.3 \\
(29.3 \\
37.5)\end{array}$ & $\begin{array}{l}21.8 \\
(18.7 \\
25.3)\end{array}$ & $\begin{array}{l}31.4 \\
(27.5 \\
35.6)\end{array}$ & $\begin{array}{l}93.8(91.4 \\
95.6)\end{array}$ \\
\hline
\end{tabular}




\begin{tabular}{|c|c|c|c|c|}
\hline Variables & Smoking & $\begin{array}{l}\mathrm{BMI} \geq 30 \\
\mathrm{~kg} / \mathrm{m}^{2}\end{array}$ & Inactive & $\begin{array}{l}\text { Inadequate } \\
\text { FV intake }\end{array}$ \\
\hline $\begin{array}{l}\text { 8. Administrative \& support service } \\
\text { activities }\end{array}$ & $\begin{array}{l}16.5 \\
(13.2 \\
20.4)\end{array}$ & $\begin{array}{l}19.2 \\
(15.9 \\
23.1)\end{array}$ & $\begin{array}{l}40.1 \\
(35.4 \\
44.9)\end{array}$ & $\begin{array}{l}95.3(93.2 \\
96.7)\end{array}$ \\
\hline 9. Transportation \& Storage & $\begin{array}{l}54.1 \\
(48.5 \\
59.5)\end{array}$ & $\begin{array}{l}20.1 \\
(15.8 \\
25.3)\end{array}$ & $\begin{array}{l}27.2 \\
(22.7 \\
32.3)\end{array}$ & $\begin{array}{l}93.1(89.3, \\
95.7)\end{array}$ \\
\hline 10. Human health \& social work activities & $\begin{array}{l}11.9(8.4 \\
16.6)\end{array}$ & $\begin{array}{l}21.4 \\
(16.6 \\
27.3)\end{array}$ & $\begin{array}{l}30.9 \\
(25.6 \\
36.9)\end{array}$ & $\begin{array}{l}92.5(88.6, \\
95.2)\end{array}$ \\
\hline 11. Financial \& insurance/takaful activities & $\begin{array}{l}11.9(8.2 \\
16.9)\end{array}$ & $\begin{array}{l}16.8 \\
(12.7 \\
21.9)\end{array}$ & $\begin{array}{l}43.5 \\
(37.5 \\
49.8)\end{array}$ & $\begin{array}{l}95.7(92.0 \\
97.8)\end{array}$ \\
\hline $\begin{array}{l}\text { 12. Professional, scientific and technical } \\
\text { activities }\end{array}$ & $\begin{array}{l}22.8 \\
(17.6 \\
28.9)\end{array}$ & $\begin{array}{l}16.8 \\
(12.4 \\
22.5)\end{array}$ & $\begin{array}{l}33.3 \\
(27.6 \\
39.5)\end{array}$ & $\begin{array}{l}91.9(87.8, \\
94.7)\end{array}$ \\
\hline 13. Others & $\begin{array}{l}30.8 \\
(27.6 \\
34.1)\end{array}$ & $\begin{array}{l}19.1 \\
(16.7 \\
21.8)\end{array}$ & $\begin{array}{l}30.8 \\
(27.7 \\
33.9)^{\prime}\end{array}$ & $\begin{array}{l}96.6(95.2 \\
97.7)\end{array}$ \\
\hline \multicolumn{5}{|l|}{ Working Sector } \\
\hline Government \& semigovernment & $\begin{array}{l}23.0 \\
(20.5 \\
25.7)\end{array}$ & $\begin{array}{l}22.7 \\
(20.3 \\
25.4)\end{array}$ & $\begin{array}{l}33.9 \\
(31.2 \\
36.7)\end{array}$ & $\begin{array}{l}92.4(90.1 \\
94.2)\end{array}$ \\
\hline Private & $\begin{array}{l}32.0 \\
(30.1, \\
33.9)\end{array}$ & $\begin{array}{l}15.8 \\
(14.5, \\
17.1)\end{array}$ & $\begin{array}{l}29.3 \\
(27.6 \\
31.0)\end{array}$ & $\begin{array}{l}95.1(94.2 \\
95.9)\end{array}$ \\
\hline Self-employed & $\begin{array}{l}36.1 \\
(33.8 \\
38.5)\end{array}$ & $\begin{array}{l}17.7 \\
(16.0 \\
19.5)\end{array}$ & $\begin{array}{l}22.6 \\
(20.7 \\
24.8)\end{array}$ & $\begin{array}{l}93.1(91.8 \\
94.2)\end{array}$ \\
\hline \multicolumn{5}{|l|}{ Working time system } \\
\hline Regular working time & $\begin{array}{l}26.0 \\
(24.4 \\
27.8)\end{array}$ & $\begin{array}{l}18.2 \\
(16.8 \\
19.5)\end{array}$ & $\begin{array}{l}32.0 \\
(30.4 \\
33.7)\end{array}$ & $\begin{array}{l}94.1(93.0 \\
95.0)\end{array}$ \\
\hline Extended hours & $\begin{array}{l}38.8 \\
(34.8 \\
42.9)\end{array}$ & $\begin{array}{l}16.8 \\
(14.2 \\
19.7)\end{array}$ & $\begin{array}{l}21.9 \\
(19.8 \\
24.1)\end{array}$ & $\begin{array}{l}94.3(92.4 \\
95.7)\end{array}$ \\
\hline Staggered days & $\begin{array}{l}41.0 \\
(38.5, \\
43.6)\end{array}$ & $\begin{array}{l}15.5 \\
(13.9 \\
17.3)^{\prime}\end{array}$ & $\begin{array}{l}23.3 \\
(20.0 \\
27.0)\end{array}$ & $\begin{array}{l}94.2(92.9 \\
95.3)\end{array}$ \\
\hline Shift hours \& part-time & $\begin{array}{l}31.4 \\
(27.9, \\
35.1)\end{array}$ & $\begin{array}{l}17.6 \\
(14.8, \\
20.6)\end{array}$ & $\begin{array}{l}28.7 \\
(25.7 \\
31.9)\end{array}$ & $\begin{array}{l}94.7(93.0 \\
96.0)\end{array}$ \\
\hline
\end{tabular}


Table 3 shows the prevalence odds ratio (POR) of seven CVD risks predicted by the sociodemographic profiles and occupational characteristics of working people in Malaysia. Older working people were more likely to have a higher prevalence of diabetes, hypertension, hypercholesterolemia, obesity, and being physically inactive. Males were associated with higher odds of developing hypertension and smoking, while females were more likely to have hypercholesterolemia and obesity and to be physically inactive. The younger age group, males and those with lower educational status were associated with a higher prevalence of smoking. In terms of main job title, professional groups, clerical workers and services \& sales workers were less likely to smoke. By industry, workers from agriculture, forestry \& fishing and transportation \& storage were more likely to smoke, while those in the education industry were less likely to smoke.

Physically inactive was prevalent among those who work as the manager and senior officials, clerical workers and services \& sales workers. In contrast, workers from agriculture, forestry \& fishing and construction were physically active. This study found that hypertension was associated with workers in the financial \& insurance/takaful activities industry. The odds of having diabetes, hypercholesteremia and obesity were not significant according to the main job title and industry classification. For work sectors, government \& semi-government workers were more likely to have a higher prevalence of diabetes, hypertension, hypercholesterolemia, smoking, and obesity than those working in the private sector. Selfemployed workers were associated with diabetes and obesity. This study found the association of those who work in extended hours to be more physically active than the workers with regular working hours. 
Table 3

The prevalence adjusted odds ratio (aPOR) of CVD risk factors among working people via household survey in Malaysia.

\begin{tabular}{|c|c|c|c|}
\hline Variables & $\begin{array}{l}\text { Diabetes } \\
\text { mellitus }\end{array}$ & Hypertension & Hypercholesterol \\
\hline & $\begin{array}{l}\text { aPOR }(95 \% \\
\mathrm{Cl}) \#\end{array}$ & $\begin{array}{l}\text { aPOR }(95 \% \\
\mathrm{Cl}) \#\end{array}$ & aPOR $(95 \% \mathrm{Cl}) \#$ \\
\hline
\end{tabular}

\section{Age group (years)}

$\begin{array}{llll}18-34 & 1.00 & 1.00 & 1.00 \\ 35-49 & 2.05(1.72, & 2.81(2.44, & 2.44(2.18,2.73) \star \star \star \\ & 2.43) \star \star \star & 3.22) \star \star \star & \\ 50 \text { and above } & 3.90(3.25, & 5.91(5.04, & 3.65(3.15,4.23) \star \star \star \\ & 4.68) \star \star \star & 6.93) \star \star \star & \end{array}$

\section{Gender}

Male

NS

$1.38(1.22$

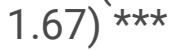

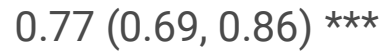

Female

1.00

1.00

1.00

\section{Level of education}

\section{Primary}

Secondary

Tertiary

Main job title classification

\section{(MASCO 2013)}

G1. Managers \& senior officials

G2. Professionals

G3. Technician \& associate professionals

G4. Clerical workers

G5. Services \& sales workers

G6. Skilled workers

G7. Unskilled or general workers Industry classification (MISC 2008)
NS

NS

1.00
NS

NS

1.00
NS

NS

1.00

\#Adjusted with all the variables in the first column, NS = not significant, $1.00=$ reference group $p$-value $<0.05^{*}, p$-value $<0.005^{* *}, p$-value $<0.0005^{* * *}$ 


\begin{tabular}{|c|c|c|c|}
\hline Variables & $\begin{array}{l}\text { Diabetes } \\
\text { mellitus }\end{array}$ & Hypertension & Hypercholesterolemia \\
\hline 1. Manufacturing & NS & NS & NS \\
\hline $\begin{array}{l}\text { 2. Wholesale \& retail trade; repair of motor } \\
\text { vehicles \& motorcycles }\end{array}$ & NS & NS & NS \\
\hline 3. Agriculture, forestry \& fishing & NS & NS & NS \\
\hline 4. Construction & NS & NS & NS \\
\hline $\begin{array}{l}\text { 5. Accommodation \& food service } \\
\text { activities }\end{array}$ & NS & NS & NS \\
\hline 6. Education & NS & NS & NS \\
\hline $\begin{array}{l}\text { 7. Public administration \& defence; } \\
\text { compulsory social security }\end{array}$ & NS & NS & NS \\
\hline $\begin{array}{l}\text { 8. Administrative \& support service } \\
\text { activities }\end{array}$ & NS & NS & NS \\
\hline 9. Transportation \& Storage & NS & NS & NS \\
\hline 10. Human health \& social work activities & NS & NS & NS \\
\hline $\begin{array}{l}\text { 11. Financial \& insurance/takaful } \\
\text { activities }\end{array}$ & NS & $\begin{array}{l}1.59(1.02 \\
2.48) \star\end{array}$ & NS \\
\hline $\begin{array}{l}\text { 12. Professional, scientific and technical } \\
\text { activities }\end{array}$ & 1.00 & 1.00 & 1.00 \\
\hline 13. Others & NS & NS & NS \\
\hline \multicolumn{4}{|l|}{ Working Sector } \\
\hline Government \& semigovernment & $\begin{array}{l}1.34(1.05 \\
1.65) \star\end{array}$ & $\begin{array}{l}1.36(1.12 \\
1.65) \star \star\end{array}$ & 1. $31(1.08,1.58)$ * \\
\hline Private & 1.00 & 1.00 & 1.00 \\
\hline Self-employed & $\begin{array}{l}1.29(1.06 \\
1.56) *\end{array}$ & NS & NS \\
\hline \multicolumn{4}{|l|}{ Working time system } \\
\hline Regular working time & 1.00 & 1.00 & 1.00 \\
\hline Extended hours & NS & NS & NS \\
\hline Staggered days & NS & NS & NS \\
\hline
\end{tabular}

\#Adjusted with all the variables in the first column, $N S=$ not significant, $1.00=$ reference group $p$-value $<0.05^{*}, p$-value $<0.005^{* *}, p$-value $<0.0005^{* * *}$ 


\begin{tabular}{|llll|}
\hline Variables & $\begin{array}{l}\text { Diabetes } \\
\text { mellitus }\end{array}$ & Hypertension & Hypercholesterolemia \\
\hline Shift hours \& part-time & NS & NS & NS \\
\hline \#Adjusted with all the variables in the first column, NS = not significant, $1.00=$ reference group \\
\hline$p$-value $<0.05^{*}, p$-value $<0.005^{* *}, p$-value $<0.0005^{* * *}$ & \\
\hline
\end{tabular}


Table 3

The prevalence adjusted odds ratio (aPOR) of CVD risks among working people via household survey in Malaysia. (cont'd)

\begin{tabular}{|c|c|c|c|c|}
\hline Variables & Smoking & $\begin{array}{l}\mathrm{BMI} \geq 30 \\
\mathrm{~kg} / \mathrm{m}^{2}\end{array}$ & $\begin{array}{l}\text { Physically } \\
\text { inactive }\end{array}$ & $\begin{array}{l}\text { Inadequate } \\
\text { FV intake }\end{array}$ \\
\hline & $\begin{array}{l}\text { aPOR }(95 \% \\
\mathrm{Cl}) \#\end{array}$ & $\begin{array}{l}\text { aPOR }(95 \% \\
\mathrm{Cl}) \#\end{array}$ & $\begin{array}{l}\text { aPOR }(95 \% \\
\mathrm{Cl}) \#\end{array}$ & $\begin{array}{l}\text { aPOR }(95 \% \\
\mathrm{Cl}) \#\end{array}$ \\
\hline \multicolumn{5}{|l|}{ Age group (years) } \\
\hline $18-34$ & 1.00 & 1.00 & 1.00 & 1.00 \\
\hline $35-49$ & $\begin{array}{l}0.83(0.72 \\
0.97) \star\end{array}$ & $\begin{array}{l}1.56(1.35 \\
1.82) * \star \star\end{array}$ & NS & NS \\
\hline 50 and above & $\begin{array}{l}0.42(0.36 \\
0.49) * \star \star\end{array}$ & $\begin{array}{l}1.57(1.29 \\
1.91)^{\star \star \star}\end{array}$ & $\begin{array}{l}1.17(1.00 \\
1.34) \star\end{array}$ & $\begin{array}{l}0.65(0.49 \\
0.87) \star \star\end{array}$ \\
\hline \multicolumn{5}{|l|}{ Gender } \\
\hline Male & $\begin{array}{l}47.3(31.6 \\
71.1)^{\star * \star}\end{array}$ & $\begin{array}{l}0.80(0.68 \\
0.93) * \star\end{array}$ & $\begin{array}{l}0.74(0.65 \\
0.80) \star \star \star *\end{array}$ & NS \\
\hline Female & 1.00 & 1.00 & 1.00 & 1.00 \\
\hline \multicolumn{5}{|l|}{ Level of education } \\
\hline Primary & $\begin{array}{l}2.50(1.98 \\
3.17) \star \star \star\end{array}$ & NS & $\begin{array}{l}0.76(0.62 \\
0.93)^{\star}\end{array}$ & NS \\
\hline Secondary & $\begin{array}{l}2.20(1.83 \\
2.64)^{\star \star \star}\end{array}$ & NS & NS & NS \\
\hline Tertiary & 1.00 & 1.00 & 1.00 & 1.00 \\
\hline \multicolumn{5}{|l|}{ Main job title classification } \\
\hline \multicolumn{5}{|l|}{ (MASCO 2013) } \\
\hline G1. Managers \& senior officials & NS & NS & $\begin{array}{l}1.60(1.20 \\
2.12)^{\star \star}\end{array}$ & NS \\
\hline G2. Professionals & $\begin{array}{l}0.73(0.55 \\
0.95) *\end{array}$ & NS & NS & NS \\
\hline $\begin{array}{l}\text { G3. Technician \& associate } \\
\text { professionals }\end{array}$ & NS & NS & NS & NS \\
\hline G4. Clerical workers & $\begin{array}{l}0.45(0.31 \\
0.65) \star \star \star\end{array}$ & NS & $\begin{array}{l}1.38(1.09 \\
1.76) *\end{array}$ & NS \\
\hline
\end{tabular}

\# Adjusted with all the variables in the first column, NS = not significant, $1.00=$ reference group $p$-value $<0.05^{*}, p$-value $<0.005^{* *}, p$-value $<0.0005^{* * *}$ 


\begin{tabular}{|c|c|c|c|c|}
\hline Variables & Smoking & $\begin{array}{l}\mathrm{BMI} \geq 30 \\
\mathrm{~kg} / \mathrm{m}^{2}\end{array}$ & $\begin{array}{l}\text { Physically } \\
\text { inactive }\end{array}$ & $\begin{array}{l}\text { Inadequate } \\
\text { FV intake }\end{array}$ \\
\hline G5. Services \& sales workers & $\begin{array}{l}0.63(0.50 \\
0.80) \star \star \star\end{array}$ & NS & $\begin{array}{l}1.25(1.01 \\
1.54) *\end{array}$ & NS \\
\hline G6. Skilled workers & NS & NS & NS & NS \\
\hline G7. Unskilled or general workers & 1.00 & 1.00 & 1.00 & 1.00 \\
\hline \multicolumn{5}{|l|}{ Industry classification (MISC 2008) } \\
\hline 1. Manufacturing & NS & NS & NS & NS \\
\hline $\begin{array}{l}\text { 2. Wholesale \& retail trade; repair of } \\
\text { motor vehicles \& motorcycles }\end{array}$ & NS & NS & NS & NS \\
\hline 3. Agriculture, forestry \& fishing & $\begin{array}{l}1.53(1.02 \\
2.28) *\end{array}$ & NS & $\begin{array}{l}0.43(0.30 \\
0.63) \star \star \star\end{array}$ & NS \\
\hline 4. Construction & NS & NS & $\begin{array}{l}0.61(0.43 \\
0.87) \star \star\end{array}$ & $\begin{array}{l}2.07(1.05 \\
4.09) *\end{array}$ \\
\hline $\begin{array}{l}\text { 5. Accommodation \& food service } \\
\text { activities }\end{array}$ & NS & NS & NS & NS \\
\hline 6. Education & $\begin{array}{l}0.44(0.26 \\
0.74) *\end{array}$ & NS & NS & NS \\
\hline $\begin{array}{l}\text { 7. Public administration \& defence; } \\
\text { compulsory social security }\end{array}$ & NS & NS & NS & NS \\
\hline $\begin{array}{l}\text { 8. Administrative \& support service } \\
\text { activities }\end{array}$ & NS & NS & NS & NS \\
\hline 9. Transportation \& Storage & $\begin{array}{l}1.67(1.10 \\
2.54) *\end{array}$ & NS & NS & NS \\
\hline $\begin{array}{l}\text { 10. Human health \& social work } \\
\text { activities }\end{array}$ & NS & NS & NS & NS \\
\hline $\begin{array}{l}\text { 11. Financial \& insurance/takaful } \\
\text { activities }\end{array}$ & NS & NS & NS & NS \\
\hline $\begin{array}{l}\text { 12. Professional, scientific and technical } \\
\text { activities }\end{array}$ & 1.00 & 1.00 & 1.00 & 1.00 \\
\hline 13. Others & NS & NS & NS & $\begin{array}{l}2.12(1.18 \\
3.81) *\end{array}$ \\
\hline
\end{tabular}

\# Adjusted with all the variables in the first column, NS = not significant, $1.00=$ reference group $p$-value $<0.05^{*}, p$-value $<0.005^{* *}, p$-value $<0.0005^{* * *}$ 


\begin{tabular}{|c|c|c|c|c|}
\hline Variables & Smoking & $\begin{array}{l}\mathrm{BMI} \geq 30 \\
\mathrm{~kg} / \mathrm{m}^{2}\end{array}$ & $\begin{array}{l}\text { Physically } \\
\text { inactive }\end{array}$ & $\begin{array}{l}\text { Inadequate } \\
\text { FV intake }\end{array}$ \\
\hline Government \& semigovernment & $\begin{array}{l}1.62(1.00 \\
1.59) \text { * }\end{array}$ & $\begin{array}{l}1.35(1.04 \\
1.75) \text { * }\end{array}$ & NS & NS \\
\hline Private & 1.00 & 1.00 & 1.00 & 1.00 \\
\hline Self-employed & NS & $\begin{array}{l}1.43(1.17 \\
1.75) \star \star\end{array}$ & NS & $\begin{array}{l}0.70(0.53 \\
0.93)^{\star}\end{array}$ \\
\hline \multicolumn{5}{|l|}{ Working time system } \\
\hline Regular working time & 1.00 & 1.00 & 1.00 & 1.00 \\
\hline Extended hours & NS & NS & $\begin{array}{l}0.81(0.65 \\
0.99)^{*}\end{array}$ & NS \\
\hline Staggered days & NS & NS & NS & NS \\
\hline Shift hours \& part-time & NS & NS & NS & NS \\
\hline \multicolumn{5}{|c|}{ \# Adjusted with all the variables in the first column, $N S=$ not significant, $1.00=$ reference group } \\
\hline \multicolumn{5}{|c|}{$p$-value $<0.05^{*}, p$-value $<0.005^{* *}, p$-value $<0.0005^{* * *}$} \\
\hline
\end{tabular}

\section{Discussion}

This study used household survey data to estimate the burden of CVD risks among working people in Malaysia aged 18-64 years, representing 12.9 million working people. This figure may reflect the working population as estimated by the Department of Statistics Malaysia in 2015 [7]. The occupational categorization can be simplified into two categories of white and blue-collar workers. The white collar refers to the professional, educated, office environment, managerial and administrative task of work and usually earning a higher income level [28]. However, this classification is too general, as the industries have both types of workers. This study utilized a newly developed questionnaire that was developed for NHMS 2015 survey. The questionnaire collected detailed occupational characteristics, which included main job title, industry classification, working sector, working time and duration of working. The industry classification is limited to 13 groups instead of 21 groups according to local guideline MISC 2008, which need further improvement in a future survey [25].

A similar occupational characteristics classification was conducted in Australia, but the study used 19 groups of industry classification [29]. There were scarce data on the burden of CVDs among working people in Malaysia using a large sample of respondents. A local study from a Malaysian cohort population estimated the prevalence of type 2 diabetes, hypercholesterolemia, and obesity according to 21 industry classifications; however, the study recruited participants between 2006 and 2012 [30]. Thus, this study focused on estimating seven CVD risks among working people using a recent national household population in 2015. Theoretically, the prevalence of CVD risks among working people should 
be lower than that among the general population because they are younger than the general population. It was true for diabetes, hypertension and physical inactivity, but working people had a higher prevalence of current tobacco smokers and an almost similar prevalence of obesity and hypercholesterolemia [17], [31]. Many studies have described increasing trends in diseases or risk factors that may contribute to CVD in low-income countries, but developed countries also face a similar pattern, but mortality is much lower than that in low-income countries $[17,24,32,33]$.

According to age categories, working people aged 50-64 years had higher POR for diabetes, hypertension, and hypercholesterolemia, but tobacco smokers were more likely among younger age working people. The higher prevalence of smoking among younger age groups may contribute to more young working people developing sudden attacks of ischemic heart disease. Following this, an early screening of CVD was recommended as early as 30 years $[3,6]$. Younger age with CVD may increase sick leave usage and affect work quality and productivity. In terms of sex, this study found that males were more likely to have hypertension and smoked tobacco. The concurrent findings had the same results as those of previous studies [18, 34]. Females had higher odds of developing hypercholesterolemia and obesity and being physically inactive, which may contribute to a higher risk of CVD at later ages [35].

Current smoking alone is the strongest risk factor for CVDs and is among the modifiable CVD risks [6]. This study found that tobacco smokers were associated with male gender, low educational status, and unskilled or general workers. By industry, smoking was associated with agriculture, forestry $\&$ fishing and transportation \& storage workers. These findings show that smoking was prevalent among blue collar workers due to many possible reasons, such as a lack of awareness of CVD, abundant supply or maladaptive behaviour towards job stress, less effort-reward imbalance and less social support at work [36-38]. Low educational status was only associated with a higher prevalence of tobacco smokers, but educational status was not associated with the likelihood of another six CVD risks in this study.

Nevertheless, the other CVD risks, such as obesity, hypercholesterolemia, diabetes and hypertension, were equally prevalent irrespective of their educational level. Education level cannot predict the increased prevalence of other CVD risks except in current tobacco smokers. It may contribute to many other factors, such as work culture, family history, genetic factors and peer influences [39, 40].

This study found that the transportation \& storage industry had a significantly higher probability of smoking. Some other studies found that transportation \& storage workers had higher odds of unideal cardiovascular health metrics, as they have a higher prevalence of physical inactivity, higher BMI, smoking and hypertension [41,42]. This group also had the highest prevalence of diabetes and a higher prevalence of hypertension, smoking, and obesity than workers in another 12 groups of new industrial classification. In terms of POR, transportation and storage workers were associated with smoking but were not associated with the odds of having diabetes, hypertension and obesity. A previous local study found that those working in transportation \& storage had higher odds of developing diabetes mellitus, but the study did not examine smoking status [30]. The mentioned study used a population of 35 to 65 years old, which contributes to their findings. Smoking was associated with transportation and storage workers because of the maladaptive behaviour in facing job demands [43].

Page $23 / 29$ 
In terms of working sectors, the government \& semigovernment sector workers had higher odds of multiple CVD risks, such as diabetes, hypertension, hypercholesterolemia, smoking and obesity, as found in previous studies $[18,33,44]$. There are many possible reasons for this finding, such as older age and sedentary working. Furthermore, government servants may be less likely to use private facilities for joining any screening program due to expensive fees. A local study noted higher odds of nonparticipation in community health screening programmes among those working in the private sector [13]. Thus, a workplace intervention program is important to reduce the burden of CVD among working people [45].

\section{Strengths and limitations}

This study utilized a nationally representative sample of the Malaysian population and applied population weightage for better estimation. One of the limitations in this study was that industry classification might need revision because the highest proportion came from "Other industry", meaning that other than the listed industry classification. In addition, the study design was a cross-sectional survey that can only estimate the current status of CVD risks among working people in Malaysia but lacks a temporality relationship in assessing the association between the independent and dependent variables.

\section{Conclusion}

This study highlighted the pattern of CVD risks according to sociodemographic profiles and occupational characteristics of working people in Malaysia via a household survey. Older age and gender have a strong association with the probability of getting CVD risks, while education level was only associated with smoking and physical activity status. The likelihood of getting diabetes, hypercholesterolemia, and obesity was not associated with the main job title and industry classification. Government \& semigovernment sector workers had higher odds of multiple CVD risks, such as diabetes, hypertension, hypercholesterolemia, smoking and obesity, than private sector workers. A holistic approach targeting those with unhealthy lifestyles and promoting a healthy workplace along with encouraging commited employers may decrease the CVD risk burden to maintain workers' productivity.

\section{Abbreviations}

aOR: adjusted odds ratios;

CVD: cardiovascular disease;

Cl: confidence interval;

DALYs: disability-adjusted life-years;

EBs: enumeration blocks; 
LQs: living quarters;

FV: fruit and vegetable;

NHMS: National Health and Morbidity Survey;

MASCO: Malaysia Standard Classification of Occupations;

MISC: Malaysia Standard Industrial Classification;

NCDs: noncommunicable diseases;

POR: prevalence odds ratio;

WRD: work-related disease.

\section{Declarations}

Ethics approval and consent to participate: This survey obtained approval from the Medical Research and Ethics Committee (MREC), Ministry of Health and was registered with the National Medical Research Registration with NMRR ID-14-1064-21877. Written consent was obtained from each individual respondent prior to data collection. This study was conducted in accordance with the Declaration of Helsinki.

Consent for publication: Not applicable

Availability of data and materials: The dataset for this study is available upon request. The main author kept the dataset according to the National Institutes of Health Malaysia research data repository guidelines.

Competing interests: The authors declare that they have no competing interests.

Funding: This work was supported by grants from the Ministry of Health Malaysia, where the grant number is under the project number NMRR ID-14-1064-21877, MFMY is the principal investigator of the grant.

Author contributions: MFMY, LKK, RMR and SMA contributed to the design of the study, data cleaning, data analysis, and interpretation of the findings. SMA, NSS, TATL and MM drafted the manuscript. Finally, the paper is reviewed and criticized by MFMY, LKK, RMR and MM. Finally, all authors approved the final version.

Acknowledgements: The authors would like to thank the Director General of Health, Malaysia for his permission to use the data from the NHMS 2015 and to publish this paper. The authors would also like to express their sincere thanks to the National Health of Institutes $(\mathrm{NIH})$ for their cooperation and assistance. 


\section{References}

1. World Health Organization. Cardiovascular diseases (CVDs); World Health Organization fact sheets [Internet]. World Health Organization. 2021 [cited 2021 Dec 18]. Available from: https://www.who.int/en/news-room/fact-sheets/detail/cardiovascular-diseases-(cvds)

2. Abbafati $C$, Abbas KM, Abbasi-Kangevari M, Abd-Allah F, Abdelalim A, Abdollahi M, et al. Global burden of 369 diseases and injuries in 204 countries and territories, 1990-2019: a systematic analysis for the Global Burden of Disease Study 2019. Lancet. 2020;396(10258):1204-22.

3. W.A Wan Ahmad (Ed). Annual Report of the Percutaneous Coronary Intervention (PCl) Registry 2017-2018. National Cardiovascular Disease Database. Kuala Lumpur, Malaysia: National Heart Association of Malaysia (NHAM); 2021. 7 p.

4. Institute for Public Health. National Health and Morbidity Survey 2011 (NHMS 2011). Vol. 1: Methodology and General Findings; 2011: 258 pages. Kuala Lumpur: Ministry of Health Malaysia; 2011.

5. Institute for Public Health. National Health and Morbidity Survey 2015 (NHMS 2015). Vol. 1: Methodology and General Findings; 2015. Kuala Lumpur, Malaysia: Ministry of Health Malaysia; 2015. 1-291 p.

6. CPG Secretariat of Health Technology Assessment Unit. Clinical Practices Guidelines on Primary \& Secondary Preventon of Cardiovascular Disease 2017 [Internet]. Putrajaya: Ministry of Health Malaysia; 2017. Available from: https://www.malaysianheart.org/?p=cpg

7. The Department of Statistics Malaysia. Labour Force Survey Report, Malaysia, 2015 [Internet]. Putrajaya; 2016. Available from: https://www.dosm.gov.my/v1/index.php? $\mathrm{r}=$ column/pdfPrev\&id=TFVqZ2NtWW9iNIJBV0pTQnZUUzBEZz09

8. Ahmed AAA, Mohammed A, Al-Shami, Jamshed S, Zawiah M, Elnaem MH, et al. Awareness of the risk factors for heart attack among the general public in pahang, malaysia: A cross-sectional study. Risk Manag Healthc Policy. 2020;13:3089-102.

9. Khoo YY, Farid NDN, Choo WY, Omar A. Prevalence, awareness, treatment and control of young-onset hypertension in Malaysia, 2006-2015. J Hum Hypertens [Internet]. 2021;2006-15. Available from: http://dx.doi.org/10.1038/s41371-020-00478-0

10. Ministry of Human Resources. Malaysia Standard Classification of Occupations (MASCO) 2013. FT Putrajaya: Ministry of Human Resources; 2013.

11. Oenning NSX, De Goulart BNG, Ziegelmann PK, Chastang JF, Niedhammer I. Associations between occupational factors and self-rated health in the national Brazilian working population. BMC Public Health. 2019;19(1):1-9.

12. Pg Ismail PK halifa., Koh D. Role of occupational health in managing non-communicable diseases in Brunei Darussalam. Glob Health Action. 2014;7:25594.

13. Kuang Kuay L, Ismail H, Ab-Majid N liana, Thamil Arasu, Saminathan Rosnah R, Ying Ying C, Aris T. Factors Associated with Non-Participation in a Health Screening Programme and its Barriers: 
Findings from the Community Empowers the Nation Programme (KOSPEN), Malaysia 2016. Int J Public Heal Res. 2020;10(1):1166-73.

14. World Health Organization. Cardiovascular diseases (CVDs) [Internet]. World Health Organization; Fact sheets. 2021 [cited 2022 Mar 28]. Available from: https://www.who.int/news-room/factsheets/detail/cardiovascular-diseases-(cvds)

15. World Health Organisation and International Labour Organisation. WHO /ILO Joint Estimates of the Work-related Burden of Disease and Injury, 2000-2016: global monitoring report. Geneva: World Health Organisation and International Labour Organisation; 2021.

16. Mawaw PM, Yav T, Mukuku O, Lukanka O, Kazadi PM, Tambwe D, et al. Prevalence of obesity, diabetes mellitus, hypertension and associated risk factors in a mining workforce, Democratic Republic of Congo. Pan Afr Med J. 2017;28:1-15.

17. McCarthy A, Damiran N. Non-Communicable Disease Risk Factors among a Cohort of Mine Workers in Mongolia. J Occup Environ Med. 2019;61(12):1072-7.

18. Manaf MRA, Nawi AM, Tauhid NM, Othman H, Rahman MRA, Yusoff HM, et al. Prevalence of metabolic syndrome and its associated risk factors among staffs in a Malaysian public university. Sci Rep [Internet]. 2021;11(1):1-11. Available from: https://doi.org/10.1038/s41598-021-87248-1

19. Faruque M, Barua L, Banik PC, Sultana S, Biswas A, Alim A, et al. Prevalence of non-communicable disease risk factors among nurses and para-health professionals working at primary healthcare level of Bangladesh: A cross-sectional study. BMJ Open. 2021;11(3):1-8.

20. Salaroli LB, Cattafesta M, Petarli GB, Ribeiro SAV, Soares AC de O, Zandonade E, et al. Prevalence and factors associated with arterial hypertension in a Brazilian rural working population. Clinics. 2020;75(6):1-7.

21. Pyakurel P, Karki P, Lamsal M, Ghimire A, Pokharel PK. Cardiovascular risk factors among industrial workers: A cross-sectional study from eastern Nepal. J Occup Med Toxicol [Internet]. 2016;11(1):1-7. Available from: http://dx.doi.org/10.1186/s12995-016-0109-6

22. Fideles IC, de Cassia Coelho de Almeida Akutsu R, Costa PRF, Costa-Souza J, Botelho RBA, Zandonadi RP. Brazilian community restaurants' low-income food handlers: Association between the nutritional status and the presence of non-communicable chronic diseases. Sustain. 2020;12(8):114.

23. Gadre V, Trivedy C. The feasibility of non-communicable disease (NCD) risk-factor estimation among forest staff at Sahyadri Tiger Reserve in Central India. Biodiversity [Internet]. 2020;21(2):97-104. Available from: https://doi.org/10.1080/14888386.2020.1793814

24. Lalla-Edward ST, Fischer AE, Venter WDF, Scheuermaier K, Meel R, Hankins C, et al. Cross-sectional study of the health of southern African truck drivers. BMJ Open. 2019;9(10):1-11.

25. Department of Statistics Malaysia. Malaysia Standard Industrial Classification 2008. 2008. 1-28 p.

26. Florescu S, Ciutan M, Sasu C, Gălăon M, Mihaescu-Pintia C. Gender and age inequalities in mental health of Romanian working adults. Eur J Public Health. 2014;24(suppl_2):322-3. 
27. Kolcic I, Polaek O, Vuletić S. Scale and dynamics of overweight and obesity epidemic in Croatia. Obes Facts. 2010;3(5):333.

28. Cheng C. Pushed to the margins: The unequal impacts of the COVID-19 crisis on marginalised Malaysian workers. Inst Strateg Int Stud Malaysia. 2020;(7):1-6.

29. Australian Bureau of Statistics. 4363.0.55.001 - Australian Health Survey: Users' Guide, 2011-13 [Internet]. 2013 [cited 2021 Nov 15]. Available from:

https://www.abs.gov.au/ausstats/abs@.nsf/Lookup/292447A4B9454E9FCA257B8D00229E9F? opendocument

30. Borhanuddin B, Ahmad N, Shah SA, Murad NAA, Zakaria SZS, Kamaruddin MA, et al. Association of job sectors with type 2 diabetes mellitus, hypercholesterolemia and obesity: A cross-sectional study from the Malaysian Cohort (TMC) project. Int Health. 2018;10(5):382-90.

31. Institute for Public Health. National Health and Morbidity Survey 2015 (NHMS 2015). Vol. II: NonCommunicable Diseases, Risk Factors \& Other Health Problems; 2015. Kuala Lumpur, Malaysia: Ministry of Health Malaysia; 2015. 1-290 p.

32. Milner K, da Silva R, Patel D, Salau S. How do we measure up? A comparison of lifestyle-related health risk factors among sampled employees in South African and UK companies. Glob Health Promot. 2018;25(1):73-81.

33. Patalen CF, Guinto SE, Atrero CT, Ducay AJD, Duante CA, Capanzana M V. Characteristics and risk factors for high fasting blood glucose among managers and government officials in the Philippines. Philipp J Sci. 2018;147(4):575-87.

34. Ahmad N, Panduragan SL, Chong Hong Soon, Gemini K, Yee San Khor, Bahrin NA, et al. Ten-year Cardiovascular Disease Risk Amongst Workers in a Tertiary Healthcare Institution in Kuala Lumpur. Borneo Epidemiol J. 2020;1(1):35-45.

35. O'Neil A, Scovelle AJ, Milner AJ, Kavanagh A. Gender/sex as a social determinant of cardiovascular risk. Circulation. 2018;137(8):854-64.

36. Lim HK, Ghazali SM, Kee CC, Lim KK, Chan YY, Teh HC, et al. Epidemiology of smoking among Malaysian adult males: Prevalence and associated factors. BMC Public Health. 2013;13(1):2-11.

37. Son SR, Choe BM, Kim SH, Hong YS, Kim BG. A study on the relationship between job stress and nicotine dependence in Korean workers. Ann Occup Environ Med [Internet]. 2016;28(1):1-9. Available from: http://dx.doi.org/10.1186/s40557-016-0113-4

38. Useche S, Cendales B, Gómez V. Work stress, fatigue and risk behaviors at the wheel: Data to assess the association between psychosocial work factors and risky driving on Bus Rapid Transit drivers. Data Br [Internet]. 2017;15:335-9. Available from: http://dx.doi.org/10.1016/j.dib.2017.09.032

39. Phing $\mathrm{CH}$, Saad HA, Yusof BNM, Taib MNM. Determinants of metabolic syndrome among Malaysian government employees. Int Med J Malaysia. 2018;17(1):87-92.

40. Abu Saad H, Low PK, Jamaluddin R, Chee HP. Level of physical activity and its associated factors among primary healthcare workers in Perak, Malaysia. Int J Environ Res Public Health. 2020;17(16):1-12. 
41. Lian Y. Stress at work in patients with cardiometabolic disease. Lancet Diabetes Endocrinol [Internet]. 2018;6(9):676-8. Available from: http://dx.doi.org/10.1016/S2213-8587(18)30172-4

42. Shockey TM, Sussell AL, Odom EC. Cardiovascular Health Status by Occupational Group - 21 States, 2013. MMWR Morb Mortal Wkly Rep. 2016;65(31):793-8.

43. Useche SA, Ortiz VG, Cendales BE. Stress-related psychosocial factors at work, fatigue, and risky driving behavior in bus rapid transport (BRT) drivers. Accid Anal Prev [Internet]. 2017;104:106-14. Available from: https://www.sciencedirect.com/science/article/pii/S0001457517301604

44. Tairea K, Kool B, Harries AD, Bissell K, Gounder S, Hill PC, et al. Characteristics of government workers and association with diabetes and hypertension in the Cook Islands. Public Heal action. 2014;4(1):S34-8.

45. World Health Organization. WHO global plan of action on workers' health (2008-2017): Baseline for implementation. 2013. Geneva: World Health Organization; 2015.

\section{Supplementary Files}

This is a list of supplementary files associated with this preprint. Click to download.

- 4A1Supplement1CVD.docx 